\title{
Interaction between vortices in models with two order parameters
}

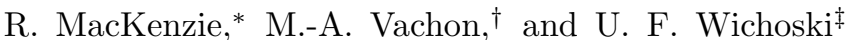 \\ Laboratoire René-J.-A.-Lévesque, Université de Montréal \\ C.P. 6128, Succ. Centre-ville, Montréal, QC H3C 3J7
}

\begin{abstract}
The interaction energy and force between widely separated strings is analyzed in a field theory having applications to superconducting cosmic strings, the $\mathrm{SO}(5)$ model of high-temperature superconductivity, and solitons in nonlinear optics. The field theory has two order parameters, one of which is broken in the vacuum (giving rise to strings), the other of which is unbroken in the vacuum but which could nonetheless be broken in the core of the string. If this does occur, there is an effect on the energetics of widely separated strings. This effect is important if the length scale of this second order parameter is longer than that of the other fields in the problem.
\end{abstract}

PACS numbers: 74.20.De,74.20.Mn,74.72.-h,11.27.+d,98.80.Cq,42.65.Tg

\section{INTRODUCTION}

In at least three very different contexts, nonlinear effects in field theories with spontaneous symmetry breaking give rise to topological solitons wherein a second order parameter (unbroken in the vacuum) attains a nonzero expectation value in the core of the soliton.

First, bosonic superconducting cosmic strings [1] arise in a model with two $U(1)$ symmetries. The first of these (which could be gauged or ungauged) is spontaneously broken by a complex scalar field $\varphi$; this gives rise to the possibility of string solutions, where the phase of $\varphi$ changes by $2 \pi$ around a large loop in space. In the core of such a string, $\varphi \rightarrow 0$. The second symmetry is gauged and unbroken, and is identified with electromagnetism. It is supposed that an electromagnetically charged scalar field $\sigma$ exists; although its VEV is zero, the potential $V(\sigma, \varphi)$ is such that if one forces $\varphi$ to zero, then $V(\sigma, 0)$ is minimized for $\sigma \neq 0$. Thus, it is possible that $\sigma$ attains an expectation value inside the core of a string, making the string a superconducting wire. Whether or not this actually occurs is a detailed dynamical question. A variety of generalizations of this idea have been discussed in Refs. [2]- 10 .

Second, in nonlinear optics, one can consider beams for which the amplitude of the electromagnetic field envelope function vanishes and its phase changes by an integer multiple of $2 \pi$ at a given point. In a nonlinear medium such a configuration can be a stable solution of the equations of motion, known as an optical vortex soliton (see [11] for references and review). By coupling a second propagating mode to the first, the vortex can act as a waveguide for the second mode, which is confined to the core of the vortex (for theoretical work, see [12, 13]; for recent experimental results see [14, 15, 16]). The first and second modes are analogous to the fields $\varphi$ and $\sigma$ above, respectively, although in the optical context both fields are ungauged. (It should also be mentioned that the fields are not really order parameters here.)

Finally, the SO(5) model of high-temperature superconductivity (HTSC) 17] is a "unification" of the two phenomena that occur in these materials at low temperature: antiferromagnetism (AF) at low doping (including the undoped case) and superconductivity (SC) at higher doping. The Ginzburg-Landau (GL) model is written in terms of a five-component real order parameter composed of a three-component field $\boldsymbol{\eta}$ describing antiferromagnetism and a two-component field $\phi$ describing superconductivity. In the SC phase, the latter field attains an expectation value, and strings (vortices) exist, just as in conventional $(\mathrm{SO}(2))$ superconductivity. It is possible that $\boldsymbol{\eta}$ attains a nonzero expectation value inside the core, making the core AF, which could provide an experimental test of the $\mathrm{SO}(5)$ model [18.

In this paper, we will study a field theory which applies (with minor modifications) to any of the above situations, and will study the energetics of two (widely-separated) vortices. In the cosmic string context this has an influence on the dynamics of a network of strings [19, 20, 21]; in optics this affects the stability of solitonic waveguides [11]; and in the superconducting case this is one way to study the type of superconductor described by a given model, which provides another test of the $\mathrm{SO}(5)$ model $[22,23]$.

\footnotetext{
*Electronic address: richard.mackenzie@umontreal.ca

${ }^{\dagger}$ Electronic address: vachon@lps.umontreal.ca

${ }^{\ddagger}$ Electronic address: wichoski@lps.umontreal.ca
} 
In the next section, we will establish notation and review previous work (expressed in the language of superconductivity, but easily translated into the other contexts), wherein the effect of the AF phase on the magnetic behaviour was studied, and the conditions under which $\mathrm{SO}(5)$ superconductors are type I or II were determined. This was done in two ways. First, the free energy $F$ of a vortex as a function of its winding number $m$ was calculated numerically 22. If the energy per unit winding number $\mathcal{F}(m) \equiv F(m) / m$ is a decreasing function of $m$, type I superconductivity results, since it is then energetically preferable for a given flux to penetrate a superconductor in one large region, inside which superconductivity is destroyed. On the other hand, if $\mathcal{F}(m)$ is an increasing function of $m$, the superconductor is type II, since the energetics then prefers a given amount of flux to be divided into a network of vortices of winding number $m=1$. Second, the type of superconductor was deduced from a determination 23. of the critical magnetic fields (see, for example, 24]). While this approach is perhaps less intuitive than the previous one, it has the advantage that a simple analytical determination of the boundary between type I and type II superconductivity can be made.

A third way of differentiating between type I and type II superconductors is through the force between two widelyseparated vortices: they attract or repel for superconductors of type I or II, respectively. This approach is very intuitive, and is also applicable to both the cosmic strings and nonlinear optics contexts (where the notion of critical magnetic fields which restore the symmetry broken in bulk doesn't apply). In this article, we will examine this third approach, adopting for the most part the language of $\mathrm{SO}(5)$ superconductivity, though much of the discussion can easily be exported to other contexts such as those mentioned above.

The method used is essentially that used by Speight [25]. We write an expression for the energy of two vortices using a point vortex approximation, wherein the full nonlinear free energy is replaced by a linearization of it plus point sources for each vortex. The approximation is expected to be valid if the intervortex separation is much larger than the core size. The approximate free energy can be written as the sum of the individual vortex energies and an interaction energy, from which the intervortex force can easily be found. In conventional SC, vortices attract or repel one another in type I and II SC, respectively. As we will see, this is not always the case here: for certain values of the parameters of the $\mathrm{SO}(5)$ model, a superconductor which is type I (in the thermodynamic sense) will have repulsive, not attractive, vortices. This unusual behaviour is unlikely to be seen in HTSC, however, as it occurs only for small values of the GL parameter $(\kappa \sim o(1))$, whereas in all known HTSCs, $\kappa \gtrsim 50$.

\section{REVIEW OF PREVIOUS WORK}

The model we wish to consider is described by the following two-dimensional free energy:

$$
\begin{gathered}
\hat{F}=\int d^{2} x\left\{\frac{(\nabla \times \hat{\mathbf{A}})^{2}}{8 \pi}+\frac{\hbar^{2}}{2 m^{*}}\left|\left(-i \nabla-\frac{e^{*}}{\hbar c} \hat{\mathbf{A}}\right) \hat{\phi}\right|^{2}\right. \\
\left.+\frac{\hbar^{2}}{2 m^{*}}(\nabla \hat{\boldsymbol{\eta}})^{2}+V(\hat{\phi}, \hat{\boldsymbol{\eta}})\right\}
\end{gathered}
$$

Here $\hat{\phi}$ and $\hat{\boldsymbol{\eta}}$ are the SC and AF order parameters, respectively. The former is a complex field associated with the $\mathrm{U}(1)$ gauge field $\hat{\mathbf{A}}$, while the latter is a real triplet whose $\mathrm{SO}(3)$ symmetry is ungauged. 29.

The potential is taken to be an even, quartic function of $|\hat{\phi}|$ and $|\hat{\boldsymbol{\eta}}|$ :

$$
V(\hat{\phi}, \hat{\boldsymbol{\eta}})=-\frac{a_{1}^{2}}{2}|\hat{\phi}|^{2}-\frac{a_{2}^{2}}{2}|\hat{\boldsymbol{\eta}}|^{2}+\frac{b}{4}\left(|\hat{\phi}|^{4}+2|\hat{\phi}|^{2}|\hat{\boldsymbol{\eta}}|^{2}+|\hat{\boldsymbol{\eta}}|^{4}\right)
$$

For simplicity, we have imposed $\mathrm{SO}(5)$ symmetry on the quartic couplings; the quadratic couplings are negative to give rise to symmetry breaking. The ground state depends on the value of the parameter $\beta \equiv a_{2}^{2} / a_{1}^{2}$, and is SC if $\beta<1$. We are primarily interested in this case; the ground state can be written $(|\hat{\phi}|,|\hat{\boldsymbol{\eta}}|)=(v, 0)$, where $v \equiv a_{1} / \sqrt{b}$.

The model can be simplified somewhat by rescaling the fields and the position variable. Defining dimensionless (unhatted) quantities

$$
\hat{\mathbf{A}}=\frac{a_{1} c \sqrt{m^{*}}}{e^{*}} \mathbf{A}, \quad \hat{\phi}=v \phi, \quad \hat{\boldsymbol{\eta}}=v \boldsymbol{\eta}, \quad \mathbf{x}=\sqrt{\frac{m^{*} c^{2}}{4 \pi e^{* 2} v^{2}}} \mathbf{s}, \quad \hat{F}=\frac{a_{1}^{2} c^{2} m^{*}}{4 \pi e^{* 2}} F,
$$

we have the dimensionless free energy

$$
\begin{gathered}
F=\frac{1}{2} \int d^{2} s\left\{(\nabla \times \mathbf{A})^{2}+\frac{1}{\kappa^{2}}\left(|(-i \nabla-\kappa \mathbf{A}) \phi|^{2}+(\nabla \boldsymbol{\eta})^{2}\right)\right. \\
\left.-\phi^{2}-\beta \eta^{2}+\frac{1}{2}\left(\phi^{2}+\eta^{2}\right)^{2}+\frac{1}{2}\right\},
\end{gathered}
$$


where $\phi=|\phi|, \eta=|\boldsymbol{\eta}|$, the derivatives are now with respect to $\mathbf{s}$, and

$$
\kappa=\sqrt{\frac{b}{4 \pi}} \frac{m^{*} c}{\hbar e^{*}}
$$

is the usual GL parameter. (A constant has been added to $F$ so that the ground state has zero energy.)

From (2), we see that the behaviour of the model is completely determined by two dimensionless parameters: $\kappa$ and the parameter $\beta$, which is the ratio of the quadratic coefficients of $\eta$ and $\phi$.

An observation which will be useful below is that if we set $\eta=0$, then the free energy (a function of $\phi$ and $\mathbf{A}$ only) reduces to that of the $\mathrm{SO}(2)$ model. Thus, for example, the energy of a static configuration for which $\eta=0$ in the $\mathrm{SO}(5)$ model will be exactly equal to the energy of the same field configuration in the $\mathrm{SO}(2)$ model with the same value of $\kappa$.

Since the complex field $\phi$ attains a nonzero VEV, vortex solutions exist, wherein the phase of $\phi$ changes by $2 \pi$ around a large circle in space. One can also consider configurations of higher winding number; a rotationally symmetric ansatz of winding number $m$ (" $m$-vortex") is essentially the conventional one for $\phi$ and A together with a rotationally invariant ansatz for $\boldsymbol{\eta}$, whose orientation is taken to be a fixed, arbitrary unit vector ê:

$$
\phi(\mathbf{s})=\varphi(s) e^{i m \theta}, \quad A_{i}(\mathbf{s})=\epsilon_{i j} \frac{s_{j}}{s} A(s), \quad \boldsymbol{\eta}(\mathbf{s})=\hat{\mathbf{e}} \eta(s) .
$$

As in the conventional case, these solutions carry a magnetic flux proportional to $m$ (specifically, the dimensionless flux is $\Phi=-2 \pi m / \kappa)$.

As mentioned in the Introduction, a non-vanishing $\eta$ field may appear inside the core of the vortex due to the competition between the potential and gradient energy terms. By examining the potential, one can see qualitatively that for fixed $\kappa$, as $\beta$ increases, the impetus for $\eta$ to be nonzero in the core of a vortex grows, since the potential energy savings gained by having $\eta \neq 0$ increases with $\beta$, while the kinetic energy cost is independent of $\beta$. Thus, we can define a critical value of $\beta$, above which the core of a vortex is AF and below which it is normal. This critical value depends upon $\kappa$, and also upon the winding number $m$ of the vortex. (The dependence on $m$ can also be argued qualitatively, by noting that the larger the winding number, the greater the region in which $\varphi$ is nearly zero, and the greater the impetus for $\eta$ to be nonzero.) $\beta_{A F}(\kappa, m)$ was found numerically for $m=1$ to 5 in Ref. [22].

The fact that the model reduces to the $\mathrm{SO}(2)$ model if $\eta=0$ enables us to easily make contact with that model; we need only set $\beta$ to zero, since then there is never any reason for $\eta$ to be nonzero in all situations we will consider here.

Substituting the ansatz (3) into (2), we find the following free energy:

$$
\begin{gathered}
F(m)=\pi \int s d s\left\{\left(A^{\prime}+\frac{A}{s}\right)^{2}+\frac{1}{\kappa^{2}}\left[\varphi^{\prime 2}+\left(\frac{m}{s}+\kappa A\right)^{2} \varphi^{2}+\eta^{\prime 2}\right]\right. \\
\left.-\varphi^{2}-\beta \eta^{2}+\frac{1}{2}\left(\varphi^{2}+\eta^{2}\right)^{2}+\frac{1}{2}\right\} .
\end{gathered}
$$

The free energy, written as a function of $m$, depends also, of course, on the two parameters of the model, $\kappa$ and $\beta$.

The equations of motion that follow from (4) are

$$
\begin{gathered}
\frac{1}{\kappa^{2}}\left[\varphi^{\prime \prime}+\frac{1}{s} \varphi^{\prime}-\left(\frac{m}{s}+\kappa A\right)^{2} \varphi\right]+\varphi\left(1-\varphi^{2}-\eta^{2}\right)=0, \\
\frac{1}{\kappa^{2}}\left(\eta^{\prime \prime}+\frac{1}{s} \eta^{\prime}\right)+\eta\left(\beta-\varphi^{2}-\eta^{2}\right)=0 \\
A^{\prime \prime}+\frac{1}{s} A^{\prime}-\frac{1}{s^{2}} A-\left(\frac{m}{k s}+A\right) \varphi^{2}=0 .
\end{gathered}
$$

These equations cannot be solved analytically; however, asymptotic solutions for large $s$ can be found. Defining

$$
\varphi(s)=1-f(s) \quad \text { and } \quad A(s)=-\frac{m}{\kappa s}+a(s),
$$

the fields $f, a$ and $\eta$ approach zero exponentially as $s \rightarrow \infty$. The linearized equations in these fields have solutions

$$
f(s)=C_{f} K_{0}(\sqrt{2} \kappa s), \quad a(s)=C_{a} K_{1}(s), \quad \eta(s)=C_{\eta} K_{0}(\sqrt{1-\beta} \kappa s)
$$




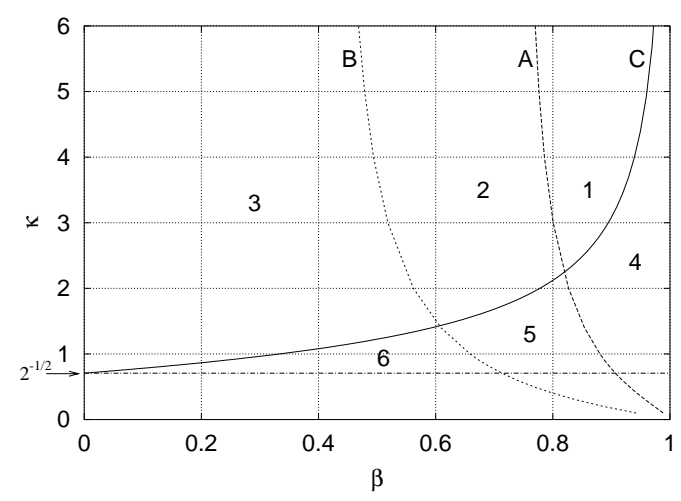

FIG. 1: Stability of vortex lattice in the SO(5) model. Curves A and B separate the normal core region (left) from the AF core region (right) in the case of $m=1$ and $m=2$ vortices, respectively. Curve $\mathrm{C}$ depicts the border between type I and type II behaviour (see (11)). These curves divide the $\beta-\kappa$ plane into sevelar regions, the most interesting of which are regions 5 and 6 , where the SC is type I yet vortices (of winding number 1) repel one another.

where $K_{0}$ and $K_{1}$ are modified Bessel functions of the second kind and the $C$ 's are constants not determined by the linear equations. These results will be useful for deriving the potential energy between a pair of widely-separated vortices in the next section.

By studying either the energetics of vortices as a function of their winding number 22] or by studying the critical magnetic fields 23], one can determine a curve in the $\beta-\kappa$ plane indicating the boundary between type I and type II behaviour, which yields a surprising result.

To see this, let us first recall the situation in the conventional $\mathrm{SO}(2)$ model, focusing our attention on vortex energetics. There, the only parameter is $\kappa$, and $\kappa=1 / \sqrt{2} \equiv \kappa_{c}$ is the critical value separating type I $\left(\kappa<\kappa_{c}\right)$ and type II $\left(\kappa>\kappa_{c}\right)$ behaviour. For example, one finds that the energy per unit magnetic flux of a vortex $\mathcal{F}(m)=F(m) / m$ decreases or increases with $m$ according to whether $\kappa<\kappa_{c}$ or $\kappa>\kappa_{c}$. In either case, $\mathcal{F}(m)$ is a monotonic function of $m$, tending towards a constant value for large $m$.

In the $\mathrm{SO}(5)$ case, the monotonicity of $\mathcal{F}(m)$ is no longer guaranteed. The reason is that for fixed $\kappa$ and $\beta$, as $m$ increases one can go from a normal vortex core to an AF core. As long as the core is normal, its energy is exactly as in the $\mathrm{SO}(2)$ model, as can be seen from (2). However, as $m$ increases, eventually the core becomes AF, at which point the energetic picture changes: in particular, the energy of all subsequent $m$-vortices will be reduced (relative to the equivalent $\mathrm{SO}(2)$ model), lessening the degree to which the superconductor is type II, and in some cases even changing the superconductor from type II to type I. For fixed $\kappa$, this effect is stronger the larger the value of $\beta$; we can therefore define a critical value $\beta_{c}(\kappa)$ as that value for which $\mathcal{F}(m)$ goes from an increasing (type II) to a decreasing (type I) function of $m$, in the limit of large $m$.

It is strange, however, that (in contrast to conventional SC) $\mathcal{F}(m)$ is not necessarily a monotonic function: it could increase with $m$ while the core is normal and decrease with $m$ subsequently. The large- $m$ behaviour indicates a type I superconductor, yet the energy of a 2-vortex is more than double that of a single vortex, which is a feature of type II superconductivity. This does not cast doubt on the fact that such a SC is indeed type I, in a thermodynamic sense; however, it will certainly affect vortex dynamics.

The behaviour at large $m$ is confirmed by studying the critical magnetic fields $H_{c}$ and $H_{c 2}$. As in conventional SC, the relative magnitude of these is an indicator of the type of SC. In [23] the critical fields were found to be given by the following expressions:

$$
H_{c}(\beta)=H_{c}^{0} \sqrt{1-\beta^{2}}, \quad H_{c 2}(\beta)=\sqrt{2} \kappa H_{c}^{0}(1-\beta),
$$

where $H_{c}^{0}$ is the thermodynamic critical field in the conventional SC model. The border between type I and type II will then be given by equating these two fields, leading to the following expressions for $\beta_{c}(\kappa)$ and, by inversion, $\kappa_{c}(\beta)$ (that value of $\kappa$ above which a SC is type II, for fixed $\beta$ ):

$$
\beta_{c}(\kappa)=\frac{2 \kappa^{2}-1}{2 \kappa^{2}+1}, \quad \kappa_{c}(\beta)=(1 / \sqrt{2}) \sqrt{(1+\beta) /(1-\beta)} .
$$

This line, as well as the lines separating the normal/AF core boundary for $m=1$ and $m=2$ vortices, lines C, A, and B respectively, are shown in Figure 1 


\section{INTERVORTEX FORCE}

As mentioned in the Introduction, a third way of determining the magnetic nature of a superconductor is through the interaction energy and force between vortices. We will study the interaction of widely-separated vortices in the $\mathrm{SO}(5)$ model. In conventional superconductivity, vortices of any separation are attractive or repulsive in the case of type I or type II superconductors, respectively, as was shown in great detail numerically by Jacobs and Rebbi 26]. We would thus expect the intervortex force in the $\mathrm{SO}(5)$ model to change from repulsive to attractive as we cross curve $\mathrm{C}$ in Figure 1 from left to right. This turns out to be not always the case. The reason is that vortex energetics does not always reflect the type of superconductor in the $\mathrm{SO}(5)$ model, as a comparison of the following two facts demonstrates. On the one hand, the type of an $\mathrm{SO}(5)$ superconductor depends critically on the AF sector (see 23]). On the other hand, when vortices have normal cores, their behaviour (their interaction energy, in particular) is blind to the AF sector of the model, as was noted in the previous section. Thus, vortices with normal cores cannot be expected to necessarily behave in the fashion dictated by the type of superconductor involved. In particular, it is possible that vortices in a type I (in the sense of bulk thermodynamic properties) superconductor repel one another.

One subtlety which arises in the interaction of vortices once their cores become AF is that while the orientation of $\boldsymbol{\eta}$ can be taken fixed in the core of any given vortex, in the absence of anisotropies, there is no reason to expect the AF cores of different vortices to be oriented in the same direction. When it becomes energetically favourable to develop an AF core, each vortex randomly selects a drection in which $\boldsymbol{\eta}$ will point. The interaction energy between vortices will depend on the angle between the two AF order parameters. As we will see, the contribution of $\boldsymbol{\eta}$ to the vortex interaction energy is most important if the two orders parameters are parallel. Our discussion will assume for the most part that this is the case.

To clarify the situation, consider the interaction of ordinary vortices (that is, vortices of winding number 1 ). The core of these vortices is normal or AF to the left or right of curve A of Figure 1 respectively. Their interaction energy will be shown to obey the following behaviour. If the core is normal (to the left of curve A), the energetics is exactly as dictated by the $\mathrm{SO}(2)$ model: attractive for $\kappa<1 / \sqrt{2}$ and repulsive for $\kappa>1 / \sqrt{2}$. If the core is AF (to the right of curve A), then (except for a very thin transition zone wherein the AF core turns on) the energetics correctly reflects the type of superconductor involved (repulsive or attractive to the left or right of curve $\mathrm{C}$ ).

If we imagine fixing $\kappa$ and increasing $\beta$, then there are three cases. First, if $\kappa<1 / \sqrt{2}$ nothing dramatic happens: for all $\beta$ the model describes type I superconductivity and vortices attract. Eventually curve A is crossed, so the vortices develop AF cores; they remain attractive, however.

Second, if $\kappa$ lies above its value at the point of intersection of curves A and $\mathrm{C}(\kappa>2.25)$, the situation is slightly more interesting: the core turns AF to the right of curve $\mathrm{A}$, but the vortices remain repulsive until curve $\mathrm{C}$ is crossed, after which the superconductor is type I and the vortices attract.

The third, intermediate case, where curve $\mathrm{C}$ is crossed before curve $\mathrm{A}$ as $\beta$ increases $(1 / \sqrt{2}<\kappa<2.25)$, is the most interesting. When curve $\mathrm{C}$ is crossed, the superconductor becomes type $\mathrm{I}$, so the vortices ought to attract. However, their cores are normal, their energetics is as in the $\mathrm{SO}(2)$ model, and they repel, since $\kappa>1 / \sqrt{2}$. This anomalous behaviour persists until curve A is crossed, when the core becomes AF and the vortices start to attract one another - the expected behaviour for a type I superconductor.

In summary, curve $\mathrm{C}$ delineates the boundary between type I (to the right) and type II (to the left) superconductivity; however, in regions 5 and 6 the superconductor has a sort of identity crisis: it is type I, but its vortices behave as type II vortices. [30]

One can also study the interaction energy of vortices of higher winding number. The behaviour is qualitatively similar to that for 1-vortices, although the region of disagreement between vortex energetics and the type of superconductor is reduced. For 2-vortices, for example, the core turns AF at a lower value of $\beta$ for any given $\kappa$ (curve $\mathrm{B}$ rather than $\mathrm{A}$ ), and consequently it is only in region 6 that one finds disagreement. This trend continues as the winding number is increased.

With this summary of the main results concluded, let us describe the calculations involved in determining the interaction energy of a pair of vortices. For arbitrary separation, an analytic solution is not possible; even numerically, the problem is extremely difficult. However, the interaction between widely separated vortices is considerably more tractable, and is clearly of interest, in that it is an important ingredient in determining the dynamics of widely separated vortices, and is normally directly related to the type of superconductor involved.

If the vortices are widely separated, one can argue that the details of the fields in the core ought to be unimportant, and we can model them by a simplifying approximation, following Speight [25]: we can consider a linearized theory with point sources added at the location of the vortices in such a way that the long-range fields produced by the point sources in the linear theory agree with those of the vortices in the original theory.

To derive the linearized theory, we must expand the free energy (2) around the asymptotic values of the fields. Before doing this, however, it is useful to eliminate the phase of $\phi$, as this degree of freedom becomes the longitudinal gauge field. Thus, we take $\phi=1-f$ real, and expand (2) in powers of $f, \mathbf{A}$ and $\boldsymbol{\eta}$, up to quadratic terms. This 
results in:

$$
F_{\text {free }}=\frac{1}{2} \int d^{2} s\left\{(\nabla \times \mathbf{A})^{2}+\mathbf{A}^{2}+\frac{1}{\kappa^{2}}(\nabla f)^{2}+2 f^{2}+\frac{1}{\kappa^{2}}(\nabla \boldsymbol{\eta})^{2}+(1-\beta) \boldsymbol{\eta}^{2}\right\} .
$$

To this, we must add couplings to sources:

$$
F_{\text {source }}=\int d^{2} s(\rho f+\boldsymbol{\sigma} \cdot \boldsymbol{\eta}+\mathbf{j} \cdot \mathbf{A}) .
$$

The equations of motion which follow from $F_{\text {free }}+F_{\text {source }}$ are

$$
\begin{gathered}
\nabla^{2} f-2 \kappa^{2} f=\kappa^{2} \rho, \\
\nabla^{2} \boldsymbol{\eta}-(1-\beta) \kappa^{2} \boldsymbol{\eta}=\kappa^{2} \boldsymbol{\sigma}, \\
\nabla^{2} \mathbf{A}-\mathbf{A}=\mathbf{j} .
\end{gathered}
$$

The sources are to be chosen to give rise to fields which coincide with the asymptotic vortex fields, after elimination of the phase of $\phi$ via a gauge transformation. These asymptotic fields are (see (9)):

$$
f(\mathbf{s})=C_{f} K_{0}(\sqrt{2} \kappa s), \quad A_{i}(\mathbf{s})=\epsilon_{i j} \frac{s_{j}}{s} C_{a} K_{1}(s), \quad \boldsymbol{\eta}(\mathbf{s})=\hat{\mathbf{e}} C_{\eta} K_{0}(\sqrt{1-\beta} \kappa s) .
$$

We require sources such that the solutions of (1416) are (17). For $f$ and $\eta$ the answer is found directly by substitution once we recognize that

$$
\left(\nabla^{2}-\mu^{2}\right) K_{0}(\mu s)=-2 \pi \delta^{2}(\mathbf{s})
$$

we find

$$
\rho(\mathbf{s})=-2 \pi \frac{C_{f}}{\kappa^{2}} \delta^{2}(\mathbf{s}), \quad \boldsymbol{\sigma}(\mathbf{s})=-2 \pi \hat{\mathbf{e}} \frac{C_{\eta}}{\kappa^{2}} \delta^{2}(\mathbf{s}) .
$$

For the gauge field, differentiation of (18) and substitution yields

$$
j_{i}(\mathbf{s})=2 \pi C_{a} \epsilon_{i j} \partial_{j} \delta^{2}(\mathbf{s}) .
$$

The vortex is now described by point sources of magnitudes $2 \pi C_{f} / \kappa^{2}$ and $2 \pi C_{\eta} / \kappa^{2}$ for $f$ and $\eta$, respectively, and an infinitesimal current loop of magnetic dipole moment $2 \pi C_{a}$ for $\mathbf{A}$.

We are now in a position to derive the interaction energy of two widely separated vortices. Suppose the vortex positions are $\mathbf{s}_{1}$ and $\mathbf{s}_{2}$. In the point vortex approximation, each vortex is described by sources of the form (1920), displaced to the position of the vortex. Linearity of the equations of motion then implies that the fields will just be the sum of the individual vortex fields, and the energy of the configuration will be given by $F_{\text {free }}+F_{\text {source }}$, with fields $(f, \boldsymbol{\eta}, \mathbf{A})=\left(f_{1}+f_{2}, \boldsymbol{\eta}_{1}+\boldsymbol{\eta}_{2}, \mathbf{A}_{1}+\mathbf{A}_{2}\right)$ and sources $(\rho, \boldsymbol{\sigma}, \mathbf{j})=\left(\rho_{1}+\rho_{2}, \boldsymbol{\sigma}_{1}+\boldsymbol{\sigma}_{2}, \mathbf{j}_{1}+\mathbf{j}_{2}\right)$, where subscript 1,2 indicates $\mathbf{s} \rightarrow \mathbf{s}-\mathbf{s}_{1,2}$. We can subtract off the energy of each vortex to obtain the following interaction energy:

$$
F_{\text {int }}=\int d^{2} s\left(\rho_{1} f_{2}+\boldsymbol{\sigma}_{1} \cdot \boldsymbol{\eta}_{2}+\mathbf{j}_{1} \cdot \mathbf{A}_{2}\right) \equiv F_{f}+F_{\eta}+F_{A} .
$$

To evaluate $F_{f}$ we simply substitute $\rho_{1}=\rho\left(\mathbf{s}-\mathbf{s}_{1}\right)$ and $f_{2}=f\left(\mathbf{s}-\mathbf{s}_{2}\right)$; we find

$$
F_{f}=\int d^{2} s\left(-2 \pi \frac{C_{f}}{\kappa^{2}} \delta^{2}\left(\mathbf{s}-\mathbf{s}_{1}\right)\right) C_{f} K_{0}\left(\sqrt{2} \kappa\left|\mathbf{s}-\mathbf{s}_{2}\right|\right)=-2 \pi \frac{C_{f}^{2}}{\kappa^{2}} K_{0}(\sqrt{2} \kappa d),
$$

where $d=\left|\mathbf{s}_{1}-\mathbf{s}_{2}\right|$ is the separation of the vortices. The same argument applies to $F_{\eta}$, yielding

$$
F_{\eta}=-2 \pi \frac{C_{\eta}^{2}}{\kappa^{2}} \hat{\mathbf{e}}_{1} \cdot \hat{\mathbf{e}}_{2} K_{0}(\sqrt{1-\beta} \kappa d)
$$




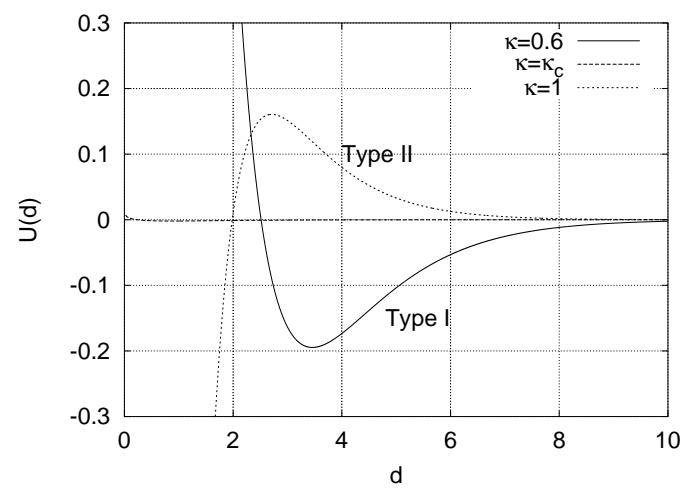

FIG. 2: Behaviour of the potential in the case of the S0(2) model $(\beta=0)$ for various values of $\kappa: \kappa=0.6$ (type I), $\kappa=\kappa_{c}$ (critical), and $\kappa=1.0$ (type II). Note that as $d$ approaches zero, the exponential behaviour is lost and the curves can not be trusted anymore.

For $F_{a}$, a similar (but slightly more complicated) procedure leads to

$$
\begin{aligned}
F_{a} & =\int d^{2} s\left(2 \pi C_{a} \epsilon_{i j} \partial_{s_{j}} \delta^{2}\left(\mathbf{s}-\mathbf{s}_{1}\right)\right) \epsilon_{i k} \frac{\left(s-s_{2}\right)_{k}}{\left|\mathbf{s}-\mathbf{s}_{2}\right|} C_{a} K_{1}\left(\left|\mathbf{s}-\mathbf{s}_{2}\right|\right) \\
& =2 \pi C_{a}^{2} \frac{\partial}{\partial s_{1 j}}\left(\frac{\left(s_{1}-s_{2}\right)_{k}}{\left|\mathbf{s}_{1}-\mathbf{s}_{2}\right|} C_{a} K_{1}\left(\left|\mathbf{s}_{1}-\mathbf{s}_{2}\right|\right)\right) \\
& =2 \pi C_{a}^{2} K_{0}(d) .
\end{aligned}
$$

Thus, the interaction energy of two widely separated vortices takes the form

$$
U(d)=2 \pi\left(C_{a}^{2} K_{0}(d)-\frac{C_{f}^{2}}{\kappa^{2}} K_{0}(\sqrt{2} \kappa d)-\frac{C_{\eta}^{2}}{\kappa^{2}} \hat{\mathbf{e}}_{1} \cdot \hat{\mathbf{e}}_{2} K_{0}(\sqrt{1-\beta} \kappa d)\right) .
$$

The first two terms give the interaction energy in the $\mathrm{SO}(2)$ case, and coincide with the results of 25, 27]. The third term is the effect of the AF cores. As can be seen, it depends on the angle between the two order parameters. If they are parallel the contribution to the interaction energy is maximally negative, resulting in the greatest attraction, while antiparallel order parameters yield a positive (repulsive) force. If the order parameters are orthogonal the AF contribution to the interaction energy is zero and the results coincide with the $\mathrm{SO}(2)$ case. Since the parallel case is the most interesting and is also energetically preferred, we will assume in what follows that the order parameters are parallel, that is, that $\hat{\mathbf{e}}_{1} \cdot \hat{\mathbf{e}}_{2}=1$.

In order to obtain useful information from (25), we must determine the constants $C_{a}, C_{f}$ and $C_{n}$ by comparing (9) with numerical solutions of the nonlinear equations (5.7), which have been obtained previously [22, 23].

We will first study the conventional $(\mathrm{SO}(2))$ case to make contact with previous work; This can be achieved within the $\mathrm{SO}(5)$ model by taking $\beta=0$. Subsequently, we will examine the general $\mathrm{SO}(5)$ case given by (25). The coefficients $C_{a}, C_{f}$ are displayed for a variety of $\kappa($ and for $\beta=0)$ in Table 1.

\begin{tabular}{|c|c|c||c|c|c|}
\hline$\kappa$ & $C_{a}$ & $C_{f} / \kappa$ & $\kappa$ & $C_{a}$ & $C_{f} / \kappa$ \\
\hline \hline 0.4 & 6.561 & 2.888 & 1.0 & 1.417 & 2.325 \\
0.5 & 4.301 & 2.635 & 1.5 & 0.822 & 2.851 \\
0.6 & 3.126 & 2.486 & 2.0 & 0.534 & 5.061 \\
$\mathbf{0 . 7 0 7}$ & $\mathbf{2 . 3 8 8}$ & $\mathbf{2 . 3 8 8}$ & 2.5 & 0.402 & 12.972 \\
0.8 & 1.969 & 2.340 & 3.0 & 0.304 & 41.688 \\
0.9 & 1.651 & 2.318 & & & \\
\hline
\end{tabular}

TABLE I: Values of $C_{a}$ and $C_{f} / \kappa$ for various values of $\kappa$ in the case where the field $\eta=0$ (achieved by setting $\beta$ to zero).

There are three cases, depending on the value of $\kappa$. The first case is $\kappa=\kappa_{c}$. At this value, the potential energy is exactly zero for all separation. Indeed, the argument of both Bessel functions takes the same value. Furthermore, 
the constants $C_{a}$ and $C_{f} / \kappa$ are equal, as can be see numerically from Table 1 and also analytically from the following argument. At $\kappa=\kappa_{c}$, we can write the free energy in a form due to Bogomol'nyi [28]; the field equations can then be written as first order differential equations:

$$
\varphi^{\prime}-\left(\frac{m}{s}+\kappa A\right) \varphi=0 \quad \text { and } \quad \kappa\left(A^{\prime}+\frac{A}{s}\right)+\frac{1}{2}\left(1-\varphi^{2}\right)=0
$$

Linearizing the first equation, we obtain $f^{\prime}=-a$; substituting (9) into that equation, we find that the second Bogomol'nyi equation is satisfied if $\sqrt{2} C_{f}=C_{a}$. This argument is only valid at $\kappa_{c}$ because (26) are not valid for other values of $\kappa$. The potential is shown in Figure 2

The second case is $\kappa>\kappa_{c}$, corresponding to (conventional) type II SC. In this case, we note that $K_{0}(\sqrt{2} \kappa d)$ falls off more rapidly than $K_{0}(d)$ so the positive term in (25) will dominate over the negative one for large enough separation, no matter what the values of the constants $C_{a}$ and $C_{f} / \kappa$. Therefore, as is well known, in conventional type II SC vortices are repulsive and give rise to stable vortex lattice. The resulting potential is displayed in Figure 2 for $\kappa=1$.

The third case is $\kappa<\kappa_{c}$, corresponding to type I SC. Here, the situation is reversed: $K_{0}(d)$ falls off more rapidly than $K_{0}(\sqrt{2} \kappa d)$, and we conclude that the long-range potential is attractive. Thus, if we start with an initial configuration formed by a number of widely-separated vortices, ultimately they will collapse into a single vortex of large winding number. This case is illustrated in Figure 2 for $\kappa=0.6$ The result (25) and the above discussion are in full agreement with [20], 25] and [26].

Let us now turn our attention to the $\mathrm{SO}(5)$ case. The coefficients $C_{a}, C_{f}$ and $C_{\eta}$ are displayed for a variety of $\kappa$ and $\beta$ in Table 2 .

\begin{tabular}{|c|c|c|c|c||c|c|c|c|c|}
\hline$\kappa$ & $\beta$ & $C_{a}$ & $C_{f} / \kappa$ & $C_{\eta} / \kappa$ & $\kappa$ & $\beta$ & $C_{a}$ & $C_{f} / \kappa$ & $C_{\eta} / \kappa$ \\
\hline \hline 1.0 & 0.000 & 1.417 & 2.325 & 0.000 & 3.0 & 0.00 & 0.362 & 41.688 & 0.000 \\
& 0.881 & 1.419 & 2.401 & 0.049 & & 0.81 & 0.362 & 45.742 & 0.083 \\
& 0.882 & 1.422 & 2.604 & 0.081 & & 0.82 & 0.364 & 52.005 & 0.119 \\
& 0.884 & 1.428 & 3.382 & 0.122 & & 0.83 & 0.364 & 61.320 & 0.145 \\
& 0.886 & 1.434 & 4.765 & 0.152 & & 0.85 & 0.368 & 74.346 & 0.185 \\
& 0.888 & 1.440 & 6.915 & 0.177 & & 0.87 & 0.371 & 88.687 & 0.216 \\
& 0.890 & 1.446 & 10.044 & 0.198 & & 0.90 & 0.379 & 132.886 & 0.249 \\
& 0.900 & 1.481 & 52.036 & 0.281 & & & & & \\
\hline
\end{tabular}

TABLE II: Values of $C_{a}, C_{f} / \kappa$, and $C_{\eta} / \kappa$ for various values of $\kappa$ and $\beta$ for $m=1$ in the case of the $\mathrm{SO}(5)$ model.

The possibility of an AF core gives rise to the surprising results mentioned at the beginning of this section. As has already been noted, if the core of the vortices is normal, there is no difference between the energetics of the $\mathrm{SO}(5)$ model and the $\mathrm{SO}(2)$ model, since the third term of (25) is zero. Thus, the interaction energy of a pair $\mathrm{SO}(5)$ vortices (of winding number 1) is unchanged from that described above, to the left of curve A in Figure 1 To the right of this curve, however, the $\eta$ field is nonzero and the third term makes an attractive contribution to the interaction force, either lessening the degree to which the vortex energetics behave in a type II manner, increasing the degree to which it behaves in a type I manner, or (the most interesting possibility) changing the vortices from repulsive to attractive.

We can read at which point this occurs from (25). The force between widely separated vortices depends on which of the fields is longest in range: the gauge field, which provides a repulsive force, or one of the scalar fields, whose forces are attractive. The range of the gauge field is 1 , while that of the $\eta$ field (the longest range of the two scalar fields, assuming it is nonzero in magnitude) is $(\sqrt{1-\beta} \kappa)^{-1}$. These forces are equal for a certain $\beta$, which we denote $\beta^{*}$ :

$$
\beta^{*}=1-1 / \kappa^{2} .
$$

The long-range vortex potential will be repulsive if $\beta<\beta^{*}$ and attractive if $\beta>\beta^{*}$.

Note that the caveat "assuming it is nonzero in magnitude" is essential: if the range of $\eta$ is longer than that of $A$, but $C_{\eta}=0$, it will obviously not have an effect on the vortex interaction.

A couple of case studies will help illustrate the situation; it is useful to see how the interaction energy varies with $\beta$ for fixed $\kappa$ (see Figure 31). In Figure 3b, the case $\kappa=3.0$ is displayed. (This corresponds to the second of the three cases mentioned near the beginning of this section.) The first two curves $(\beta=0.0$ and $\beta=0.8)$ coincide, since the core has yet to become AF (as can be ascertained from Figure 1 since these points lie to the left of Curve A). The subsequent curves show a decrease in the potential as the $\eta$ field turns on, along with its attractive contribution to 

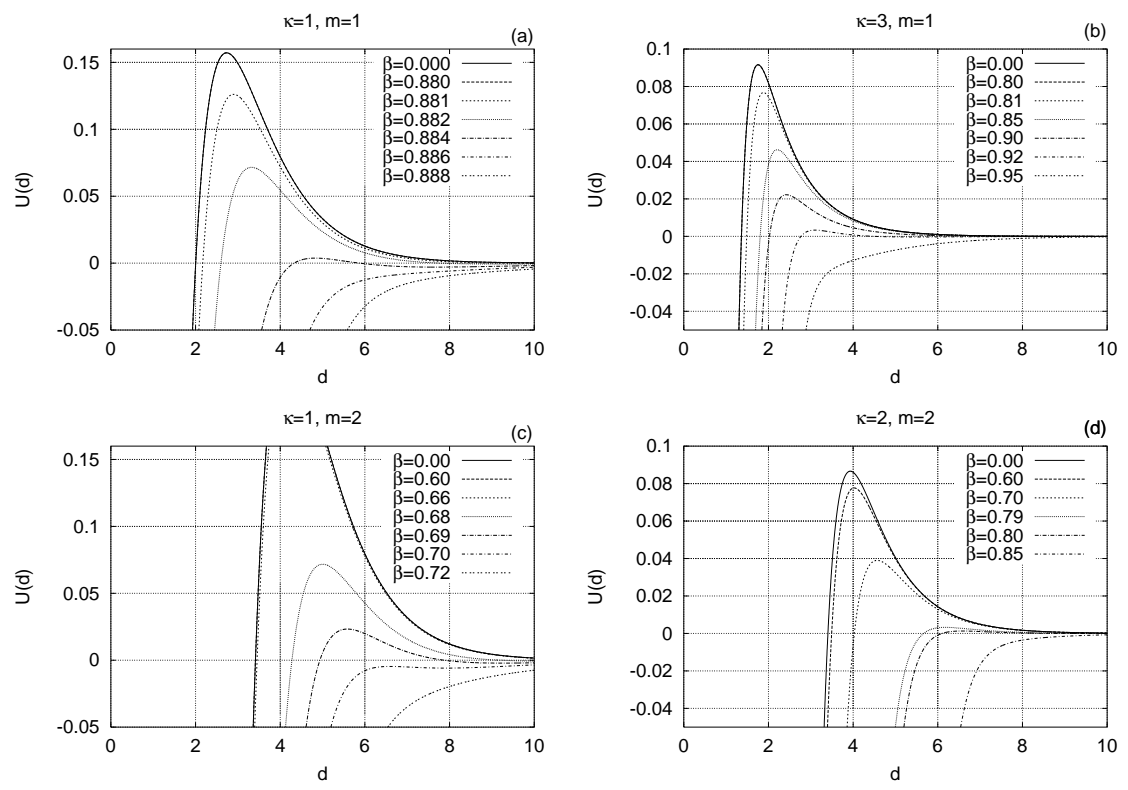

FIG. 3: Intervortex potential for $\kappa=1$ (a) and $\kappa=3$ (b) for $m=1$, and for $\kappa=1$ (c) and $\kappa=2$ (d) for $m=2$.

the vortex energy. Eventually when $\beta \simeq 0.92$, the potential goes from repulsive to attractive, in agreement with (27), and also with (11).

In Figure $3 \mathrm{k}$, the case $\kappa=1.0$ (the third case mentioned near the beginning of this section) is displayed. The first two curves, corresponding to parameters lying to the left of Curve A in Figure 1 coincide, since the core of the vortices is normal; subsequent increases in $\beta$ produce a drop in the interaction energy, as expected since $\eta$ now contributes. It is important to note, however, that for $\kappa=1.0$ the superconductor is type I starting at $\beta_{c}=0.33$. We would thus expect the vortices to attract for $\beta>0.33$. This is not the case: as can be seen from Figure 3 , the attraction begins only around $\beta \simeq 0.883$, somewhat to the right of Curve $\mathrm{A}\left(\beta_{A F}=0.881\right)$. (That it is somewhat to the right of Curve A is simply due to the fact that $\eta$ (at the center of the vortex, for example) evolves continuously with $\beta$ and is strictly zero up until Curve A; it is therefore small and will have a negligible effect on the vortex interaction until it has time to grow to an appreciable value (see the values of $C_{\eta}$ given in Table 2).) Thus, there is a zone (between curves A and C) wherein the superconductor is type I, but wherein nonetheless vortices repel one another: this is the identity crisis referred to earlier. The point is that in this region the $\eta$ field would produce an attractive long-range interaction, but it has not yet attained a nonzero value in the core and therefore has no effect on the vortex interaction.

The situation is even stranger if we consider vortices of winding number 2 , for which Curve B delineates normal (to the left) vs. $\mathrm{AF}$ (to the right) cores. For $\kappa=2.0$ (Figure $3 \mathrm{~d}$ ), the vortex turns $\mathrm{AF}$ at $\beta_{A F}=0.562$, but vortices remain repulsive until the value corresponding to the type I-type II transition $\left(\beta_{c} \simeq 0.78\right)$. For $\kappa=1.0$ (Figure 3i), the vortices develop an $\mathrm{AF}$ core at roughly $\beta_{A F}=0.659$ but remain repulsive until just to the right of Curve $\mathrm{C}$, as expected given that Curve C separates type II from type I superconductivity. However, at a lower value of $\kappa$ we would find that the interaction energy of 2-vortices in Region 6 is repulsive, which is incommensurate with the fact that the superconductor is type I there.

Note that these "identity crises" only occur for certain values of the parameters, and in particular for $\kappa<2.25$, so this does not appear to be relevant to HTSC (supposing that the $\mathrm{SO}(5)$ model provides a good description of these systems), since all known HTSCs have quite large values of $\kappa(\kappa \gtrsim 50)$. However, in the case of superconducting cosmic strings and optical vortex solitons, there are no restrictions on the value of $\kappa$ and the effect may be relevant.

It is interesting to note that the results obtained above complement those obtained in previous work where the conditions under which $\mathrm{SO}(5)$ superconductors are type I or type II were determined. In Ref. [22], the distinction between type I and type II behaviour was determined by analysing the energy per unit flux of vortices as a function of their winding number. In Ref. 23], this distinction was obtained by studying the energy density of a surface separating superconducting and normal regions at critical applied magnetic fields. The results stemming from both methods are in agreement and reveal, unexpectedly, that superconductors whose $\kappa$ values would normally be associated with type II superconductivity may in fact be type I superconductors. The surprising region is $\kappa>1 / \sqrt{2}$ (conventionally type II) and to the right of Curve $\mathrm{C}$ in Figure 1 The underlying reason is the possibility of an AF vortex core, or equivalently, the fact that an applied magnetic field induces a transition between SC and AF (not normal) phases. 


\section{CONCLUSION}

In this paper we have derived an expression for the potential energy of two widely separated vortices within the framework of a model with two order parameters, one of which is complex and attains a nonzero expectation value. Examples of physical contexts where such a situation occurs are bosonic superconducting cosmic strings, optical vortex solitons and the SO(5) model of HTSC. The approach used was a point vortex approximation, due to Speight 25]. Our starting point was a linearization of the free energy (12) to which source terms (13) were added; the form of the sources was given in (1920). The main result is the two-vortex interaction energy, (25). The constants appearing in this expression were chosen so that the long-range fields of a vortex in the point vortex approximation agree with the full nonlinear model.

The behaviour of the vortices is for the most part in agreement with previous work, described in Section 2 , namely, that for fixed $\kappa$, type II behaviour (stable vortex lattice) turns into type I behaviour (unstable lattice) for $\beta$ sufficiently close to 1 , provided that the vortices have AF cores.

This caveat, although apparently innocent, is actually quite important, and yields a surprising result. Namely, for certain regions in parameter space (regions 5 and 6 in Figure 1), the superconductor is type I, yet vortices have normal (not AF) cores and repel one another, a behaviour normally associated with type II superconductors. It would appear that in such a case a vortex lattice would be metastable rather than stable (the free energy of a macroscopic non-SC region containing the same flux being lower than that of the vortex lattice). It is ironic and amusing that the surprising behaviour uncovered in 22, 23] (type I SC for $\kappa>1 / \sqrt{2}$ ) can be thought of as being due to the fact that vortices have AF cores, whereas the surprising behaviour uncovered here (vortices which repel one another in type I $\mathrm{SC}$ ) is due to the fact that vortices have normal, not AF cores.

While the attraction or repulsion of vortices is clearly of interest in any physical situation described by the above work, the unexpected behaviour uncovered here is unlikely to be seen in HTSC, as it occurs in the wrong region of parameter space.

This work was supported by the Natural Science and Engineering Research Council of Canada and by the Fonds de Recherche sur la Nature et les technologies du Québec.

[1] E. Witten, Nucl. Phys. B249, 557 (1985).

[2] G. Lazarides and Q. Shafi, Phys. Lett. B 159, 261 (1985).

[3] E. J. Copeland, N. Turok, and M. Hindmarsh, Phys. Rev. Lett. 58, 1910 (1987).

[4] R. MacKenzie, Phys. Lett. B 197, 59 (1987).

[5] R. MacKenzie, Nucl. Phys. B303, 149 (1988).

[6] M. Hindmarsh, Phys. Lett. B 200, 429 (1988).

[7] C. T. Hill, H. M. Hodges, and M. S. Turner, Phys. Rev. D 37, 263 (1988).

[8] D. Haws, M. Hindmarsh, and N. Turok, Phys. Lett. B 209, 255 (1988).

[9] R. L. Davis and E. P. S. Shellard, Nucl. Phys. B323, 209 (1989).

[10] J. A. Frieman and B. W. Lynn, Nucl. Phys. B329, 1 (1990).

[11] Y. S. Kivshar and B. Luther-Davies, Phys. Rep. 298, 81 (1998).

[12] T. J. Alexander, Y. S. Kivshar, A. V. Buryak, and R. A. Sammut, Phys. Rev. E 61, 2042 (2000).

[13] A. H. Carlsson, J. N. Malmberg, D. Anderson, and M. Lisak, Opt. Lett. 25, 660 (2000).

[14] A. G. Truscott, M. E. J. Friese, N. R. Heckenberg, and H. Rubinsztein-Dunlop, Phys. Rev. Lett. 82, 1438 (1999).

[15] M. Morin, G. Duree, G. Salamo, and M. Segev, Opt. Lett. 20, 2066 (1995).

[16] J. U. Kang, G. I. Stegeman, and J. S. Aitchison, Opt. Lett. 20, 2069 (1995).

[17] S.-C. Zhang, Science 275, 1089 (1997), cond-mat/9610140.

[18] D. P. Arovas, A. Berlinsky, C. Kallin, and S.-C. Zhang, Phys. Rev. Lett. 79, 2871 (1997).

[19] L. M. A. Bettencourt and T. W. B. Kibble, Phys. Lett. B 332, 297 (1994), hep-ph/9405221.

[20] L. M. A. Bettencourt and R. J. Rivers, Phys. Rev. D 51, 1842 (1995), hep-ph/9405222.

[21] L. M. A. Bettencourt, P. Laguna, and R. A. Matzner, Phys. Rev. Lett. 78, 2066 (1997), hep-ph/9612350.

[22] M. Juneau, R. MacKenzie, M. A. Vachon, and J. M. Cline, Phys. Rev. B 65, 140512 (2002), cond-mat/0106172.

[23] M. Juneau, R. MacKenzie, and M. A. Vachon, Ann. Phys. 298, 421 (2002), cond-mat/0202408.

[24] M. Tinkham, Introduction to Superconductivity, 2nd edition (McGraw-Hill, 1996).

[25] J. M. Speight, Phys. Rev. D 55, 3830 (1997), hep-th/9603155.

[26] L. Jacobs and C. Rebbi, Phys. Rev. B 19, 4486 (1979).

[27] L. Kramer, Phys. Rev. B 3, 3821 (1971).

[28] E. B. Bogomol'nyi, Sov. J. Nucl. Phys. 24, 449 (1976). 
[29] Hats denote dimensionful quantities. The constants appearing in (1) are appropriate to superconductivity; readers unfamiliar with these conventions will be relieved to learn that the notation will be streamlined presently, when we go to dimensionless variables.

[30] Perhaps some clarification on what is meant by type I vs type II superconductivity is warranted. In conventional superconductivity, type II superconductivity (for instance) is characterized by $\kappa>1 / \sqrt{2}$, negative surface energy at a normal/superconducting boundary, repulsive vortices, and $H_{c 2}>H_{c}$. These are so inextricably connected that any of these features could be used as a definition of type II; the rest would follow. As we have seen, and as will be argued below (see also 23]), these features no longer imply one another in $\mathrm{SO}(5)$ superconductivity. We refer to a superconductor as type I or II according to whether the surface energy is negative or positive, or equivalently, according to the relative value of $H_{c}$ and $H_{c 2}$. 